\title{
HIGHLIGHTS
}

IMMUNOTHERAPY

\section{Alphavirus vector induces immune response in advanced cancers}

Anticancer vaccines have shown promise in treating cancers, especially those based on viral vectors; however, a limitation of viral vector-based vaccines is the induction of neutralizing antibodies that recognize viral surface proteins, which prevents repeated administration of these vaccines. Furthermore, studies have shown that the levels of $\mathrm{T}_{\mathrm{REG}}$ cells, which can impair effective immune responses, are increased in cancer patients and associated with a poor survival.

A recent study by Michael Morse and colleagues has shown that an alphavirus vector, packaged into virus-like replicon particles (VRP), can overcome the presence of neutralizing antibodies and produce potent responses in patients with advanced cancer.

First, Morse's team developed a VRP vaccine that expressed carcinoembryonic antigen (CEA), which is highly expressed in various solid tumor malignancies, and conducted preclinical studies in mice to assess immunogenicity of the vaccine. They showed an improved immune response with the vaccine in these murine models. They then went on to perform a phase I study of the vaccine, in patients with advanced cancer, and assessed immune responses against CEA by ELISA and ELISPOT. Overall, the immunizations were well tolerated.

Consistent with other published reports, the levels of $\mathrm{T}_{\mathrm{REG}}$ cells were significantly greater in cancer patients than healthy controls, but the vaccine was able to induce substantial immune responses, despite the elevated levels of immunosuppressive $\mathrm{T}_{\text {REG }}$ cells and induction of neutralizing antibodies against the VRP-based vaccine. The researchers also demonstrated that the antibodies induced by the vaccine were functional despite the presence of anti-VRP neutralizing antibodies and high $\mathrm{T}_{\mathrm{REG}}$ levels.

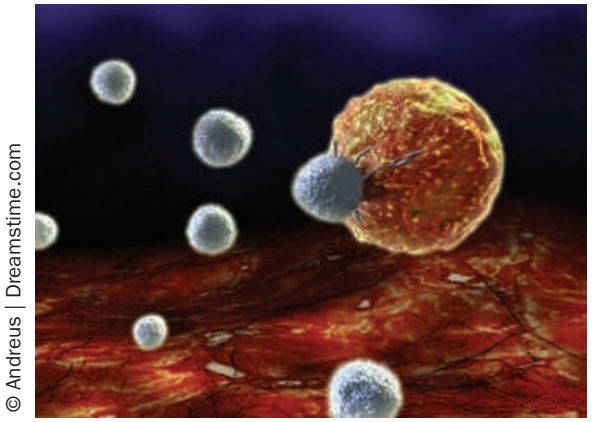

"We were able to treat advanced cancer patients with repeated immunizations with a recombinant alphavirus vector to break tolerance to nonmutated CEA despite induction of neutralizing antibody against the VRP and elevated $\mathrm{T}_{\mathrm{REG}}$ levels" explains Morse. He continues, "this is the first study we are aware of that shows a viral vector overcoming neutralizing antibodies and elevated $\mathrm{T}_{\mathrm{REGS}}$ in cancer patients".

\section{Lisa Hutchinson}

Original article Morse, M. A. et al. An alphavirus vector overcomes the presence of neutralizing antibodies and elevated numbers of Tregs to induce immune responses in humans with advanced cancer. J. Clin. Invest. 120, 3234-3241 (2010) 